\title{
Collection and Morphological Variability in Ecotypes of Indian Pennywort (Centella asiatica L.) of Hill Zone of Karnataka, India
}

\author{
C. S. Ravi ${ }^{*}$, K. Umesha ${ }^{2}$, K. HimaBindu ${ }^{3}$, G. Raviraja Shetty ${ }^{4}$ \\ and G. S. Anil Kumar \\ ${ }^{1}$ PSMAC, ZAHRS, Mudigere-577132, India \\ ${ }^{2}$ ICAR-Emeritus Professor, College of Horticulture, GKVK Post, Bengaluru-65, India \\ ${ }^{3}$ Division of Floriculture and Medicinal crops, ICAR-IIHR, Bengaluru, India \\ ${ }^{4}$ (PSMAC), College of Horticulture, Mudigere-577132, India \\ ${ }^{7}$ College of Horticulture, GKVK Post, Bengaluru-65, India
}

*Corresponding author

\section{A B S T R A C T}

\section{Keywords}

Diverse accessions, qualitative traits, variability, morphological and descriptors

Article Info

Accepted:

14 August 2019

Available Online:

10 September 2019
An investigation was carried out on exploration and collection of native germplasm of Indian pennywort for assessment and utilization of existing variability present in the population. Totally thirty nine diverse accessions were collected from 22 talukas of eight districts of hill zone of Karnataka at an altitude ranging from $363 \mathrm{~m}$ to $1064 \mathrm{~m}$ above mean sea level. The collected accessions were morphologically characterized for 14 qualitative traits as per NBPGR descriptors revealed wide variability for all the morphological traits except leaf surface. Predominantly erect plant growth habit was observed as compared to semi erect and prostrate growth habit. Majority of the accessions exhibited good regenerabilty, rosette type of leaf arrangement medium sized, orbicular shaped with dentate margin and dark green coloured leaves. Considerable differences were also noticed for petiole and flower characteristics. Majority of the accessions were of medium sized, thin petioled with pink colour pigmentation at the base. Greenish pink stolon and pink flower colours were dominant with soft textured stolonsas compared to hard textured.

\section{Introduction}

Indian Pennywort (Centella asiatica L.) is an important tropical medicinal plant belongs to the family Apiaceae with a somatic chromosome number $2 n=18$. The plant is native to South East Asian countries including India, SriLanka, China and 
Malaysia as well as SouthAfrica and has pantropical in distribution.It occurs throughout India in moist places from plains to hill ranges up to $2000 \mathrm{~m}$ (Mukherjee and Constance, 1993). The whole herb is economically important and its biological effects have been attributed to the existence of major triterpene derivatives including madecassoside, asiaticoside, madecassic andasiatic acid(Schaneberg et al., 2003). Centella asiatica $\mathrm{L}$. is a potential upcoming pharmaceutical, neutraceutical and cosmoceutical herb, gaining importance in the international trade of medicinal plants. In India, it is primarily known as "Brain food" and is often considered as "panacea" for several ailments such as antidiabetic, antimicrobial and antiproliferative properties due to the presence of triterpenoids. Indianpennywort is abundantly distributed throughout the hill zone of Karnataka and is a threatened species, as the genetic resources are markedly depleted because of it's unrestricted over exploitation from the natural habitat.

Variability assessment through morphological markers is considered to be an important step in description and characterization of germplasm. These easily observable morphological traits are useful tools for preliminary evaluation, as they offer a useful approach for assessing the extent of diversity. Hence, the present investigation was carried out to assess the magnitude of genetic diversity present in the native germplasm.

\section{Materials and Methods}

An exploration work was carried out during Kharif (July-September, 2017) in hill zone districts of Karnataka (Fig.1). The hill zone of Karnataka is situated in Western Ghats extending from Virajpete in the South (Kodagu district) to Khanapur in the North (Belgaum district). The details of accessions collected is furnished in Table 1. The stolons of each germplasm accessions (Designated as Acc.1 to Acc. 39) were collected from every taluk as one unit of exploration site from two different habitats. The collected germplasm accessions from different ecological regions were brought to ZAHRS, Mudigere and planted in nursery beds of $2 \mathrm{~m} \times 1 \mathrm{~m}$ size under poly house conditions for establishment and multiplication.

Observations on morphological characters were recorded on five randomly selected rosettes from each accession at full foliage stageby referring to NBPGR plant descriptors of Centella asiatica L. with additions as required. The plant regeneration capacity of each accessions was observed after every harvest, ability of accessions for time taken for sprouting and ground coverage were considered as criteria for categorization. The leaf size of all 40 accessions was considered visually and categorized as small, medium and large based on the visual observations. The leaf, flower and stolon colour and also petiole pigmentation at the base were recorded using RHS colour chart. The classification of accessions based on petiole length into small (less than $10 \mathrm{~cm})$, medium $(10-15 \mathrm{~cm})$ and long (more than $15 \mathrm{~cm}$ ). The list of morphological traits observed are given in Table 3.2.

\section{Results and Discussion}

\section{Collection of accessions}

A total of 39 accessions were collected from various ecological regions of Kodagu, Hassan, Chikkamagalur, Shivamogga, Uttara Kannada, Haveri, Belgaum and Dharwad districts of hill zone of Karnataka (Table 2). Maximum number of accessions were collected from five talukas of Chikkamagaluru district (11) followed by six talukas of Uttara Kannada (09), four talukas of Shivamogga (08) and three talukas of Kodagu (06). While, two 
accessions were collected from Sakaleshpura taluk of Hassan district and one accession each from Haveri, Belgaum and Dharwad district from one taluk each. Totally 22 talukas from eight districts of hill zone of Karnataka were explored at an altitude ranging from 363 $\mathrm{m}$ (Acc. 29) to $1064 \mathrm{~m}$ (Acc.11) above mean sea level for collection of the Centella asiatica L. accessions for assessment of morphological variability exists for further exploitation in crop improvement programmes.Similar exploration and collection work has been carried out by Prasad et al., (2014), Kundu et al., (2015), Singh et al., (2015), Thapa et al., (2016) and Lal et al., (2017) collected diverse genetic stocks from different locations of India in Centella asiatica.

\section{Morphological characterization of accessions}

In the present study, considerable variations were observed in plant growth habit, among 40 accessions studied, 31 accessions (Acc. 1, $2,3,4,5,6,7,8,9,10,11,12,13,14,15,17$, $18,19,20,24,25,26,27,28,29,30,34,35$, 37, 38 and Vallabh Medha) exhibited erect growth habit and six accessions exhibited (Acc. 21, 22, 23, 31, 32 and 33) semi erect growth habit. The rest of accessions (Acc. 16, 39 and 36) recorded prostrate growth habit. Since, herbage is the main economic part which is largely comprised of leaves, the accessions with erect growth are highly preferred.

Erect growing accessions are ideal than semi erect and prostate types as it allows maximum and uniform exposure to sunlight and would result in an increased dry matter production and subsequently the yield. The variations noticed in plant growth habit among the accessions are due to differences in their genetic makeup. Similar variation in different genotypes was observed by Tripathi et al., (2012) in brahmi. Among 40 accessions studied for plant regeneration capacity 16 accessions have recorded good regenerability (Acc. 1, 3, 4, 6, 7, 8, 12, 14, 18, 19, 22, 24, 28, 30, 38 and Vallabh Medha) and accessions such as Acc. 2, 5, 10, 11, 13, 15, 20, 25, 27, $29,31,33,34,35$ and 37 recorded medium regenerability. On the other hand, rest of the accessions (Acc. 9, 17, 26, 16, 36, 21, 23, 32 and 39) showed poor regeneration capacity. Centella asiatica L. is a herbaceous perennial, multiple harvests with higher biomass are possible if the accessions possess good regenerability and also due to early ground cover after every ratooning and weeds could be managed by smothering effect of the crop. The variations in ratoonability among the accessions are could be due to differences in their genetic makeup.

With regard to leaf arrangement, all the accessions exhibited rosette type except Acc. 07, 08 and Vallabh Medha which were of spreading type. The variation in leaf arrangement could be attributed purely to the influence of genetic constitution of the accessions. Kaur and Saggoo (2010) observed similar variations in leaf arrangement among Aloe vera accessions of North India.

Considerable phenotypic variations were registered for leaf size viz., small, medium and large. The leaf size determines the photosynthetic efficiency of the accessions, on which growth, yield and synthesis of secondary metabolites depends greatly.

The accessions Acc.07, 11, 12, 16, 26 and Vallabh Medha had larger leaves. While, rest of the accessions had medium sized leaves, except Acc.03 and 39 which recorded small leaves.

The variations in leaf size is expected among the accessions as the attribute is genetic in a given set of environmental conditions, as the accessions interact with the environmental conditions, specially soil moisture supply and light. 
Table.1 Details of exploration and collection districts and talukas of Centella asiatica L. germplasm accessions under hill zone of Karnataka

\begin{tabular}{|c|c|c|c|c|c|c|c|}
\hline \multirow[t]{2}{*}{ Accessions } & \multicolumn{3}{|l|}{ Collection site } & \multirow[t]{2}{*}{ Habitat } & \multicolumn{3}{|c|}{ Geo reference } \\
\hline & Village & $\begin{array}{l}\text { Mandal/Taluk/ } \\
\text { Tehsil }\end{array}$ & District & & $\begin{array}{l}\text { Latitude } \\
(\mathbf{N})\end{array}$ & $\begin{array}{l}\text { Longitude } \\
\text { (E) }\end{array}$ & $\begin{array}{l}\text { Altitude } \\
(\mathbf{m})\end{array}$ \\
\hline Acc. 1 & Halekote & Mudigere & Chikkamagaluru & Paddy field & $13^{0} .07$ & $75^{0} .37^{\prime}$ & 916 \\
\hline Acc. 2 & Kademadkal & Mudigere & Chikkamagaluru & Paddy field & $13^{0} .09^{\prime}$ & $75^{0} .40^{\prime}$ & 873 \\
\hline Acc. 3 & Heggadde & Sringeri & Chikkamagaluru & Up land arecanut & $13^{0} .27^{\prime}$ & $75^{0} .15^{\prime}$ & 558 \\
\hline Acc. 4 & Nagalapura & Koppa & Chikkamagaluru & Paddy field & $13^{0} .30^{\prime}$ & $75^{0} .18^{\prime}$ & 531 \\
\hline Acc. 5 & Kuppali & Thirthahalli & Shivamogga & Paddy field & $13^{0} .35^{\prime}$ & $75^{0} .18^{\prime}$ & 569 \\
\hline Acc. 6 & IthigeSeegodu & N.R. Pura & Chikkamagaluru & Paddy field & $13^{0} .20^{\prime}$ & $75^{0} .27^{\prime}$ & 639 \\
\hline Acc. 7 & $\begin{array}{l}\text { Kymanahalli } \\
\text { (Rakshidi Estate) }\end{array}$ & Sakaleshpura & Hassan & Coffee + Pepper plantation & $13^{0} .01^{\prime}$ & $75^{0} .4^{\prime}$ & 859 \\
\hline Acc. 8 & Kallarahalli & Sakaleshpura & Hassan & $\begin{array}{l}\text { Low land coffee }+ \\
\text { arecanut plantation }\end{array}$ & $12^{0} .56^{\prime}$ & $75^{0} .42^{\prime}$ & 820 \\
\hline Acc. 9 & Madaravalli & Somwarapet & Kodagu & Paddy field & $12^{0} .44^{\prime}$ & $75^{0} .53^{\prime}$ & 830 \\
\hline Acc. 10 & Balagunda & Somwarapet & Kodagu & Coffee + Pepper & $12^{0} .34^{\prime}$ & $75^{0} .50^{\prime}$ & 1040 \\
\hline Acc. 11 & Makandur & Madikeri & Kodagu & Coffee + Pepper & $12^{0} .27^{\prime}$ & $75^{0} .46^{\prime}$ & 1062 \\
\hline Acc. 12 & Madikeri & Madikeri & Kodagu & Paddy field & $12^{0} .25^{\prime}$ & $75^{0} .43^{\prime}$ & 1024 \\
\hline Acc. 13 & Ammathi & Virajpet & Kodagu & Coffee plantation & $12^{0} .15^{\prime}$ & $75^{0} .52^{\prime}$ & 831 \\
\hline Acc. 14 & Puliyeri & Virajpet & Kodagu & Low land Coffee + Erythrina & $12^{0} .14^{\prime}$ & $75^{0} .51^{\prime}$ & 854 \\
\hline Acc. 15 & Doona & Hosanagara & Shivamogga & Paddy field & $14^{0} .00^{\prime}$ & $75^{0} .17^{\prime}$ & 573 \\
\hline Acc. 16 & Menase & Hosanagara & Shivamogga & Forest land & $14^{0} .01^{\prime}$ & $75^{0} .10^{\prime}$ & 543 \\
\hline Acc. 17 & Henigere & Sagara & Shivamogga & Low land arecanut plantation & $14^{0} .71^{\prime}$ & $75^{0} .04^{\prime}$ & 527 \\
\hline Acc. 18 & BhramanaManchale & Sagara & Shivamogga & Forest & $14^{0} .10^{\prime}$ & $75^{0} .51^{\prime}$ & 531 \\
\hline
\end{tabular}


Table 1.Contd....

\begin{tabular}{|c|c|c|c|c|c|c|c|}
\hline \multirow[t]{2}{*}{ Accessions } & \multicolumn{3}{|l|}{ Collection site } & \multirow[t]{2}{*}{ Habitat } & \multicolumn{3}{|c|}{ Geo reference } \\
\hline & Village & $\begin{array}{l}\text { Mandal/Taluk/ } \\
\text { Tehsil }\end{array}$ & District & & $\begin{array}{l}\text { Latitude } \\
\text { (N) }\end{array}$ & $\begin{array}{l}\text { Longitude } \\
\text { (E) }\end{array}$ & $\begin{array}{l}\text { Altitude } \\
\text { (m) }\end{array}$ \\
\hline Acc. 19 & Tugur & Sorabha & Shivamogga & Low land arecanut & $14^{0} .15^{\prime}$ & $75^{0} .06^{\prime}$ & 519 \\
\hline Acc. 20 & Hosabale & Sorabha & Shivamogga & Forest & $14^{0} .19^{\prime}$ & $75^{0} .02^{\prime}$ & 529 \\
\hline Acc. 21 & Siralagi & Siddapura & Uttara Kannada & Upland arecanut & $14^{0} .20^{\prime}$ & $75^{0} .57^{\prime}$ & 486 \\
\hline Acc. 22 & Siddapura & Siddapura & Uttara Kannada & Paddy field & $14^{0} .11^{\prime}$ & $74^{0} .53^{\prime}$ & 512 \\
\hline Acc. 23 & Terakanahalli & Sirsi & Uttara Kannada & Low land arecanut & $14^{0} .36^{\prime}$ & $74^{0} .50^{\prime}$ & 619 \\
\hline Acc. 24 & Isaluru & Sirsi & Uttara Kannada & Low land banana field & $14^{0} .40^{\prime}$ & $74^{0} .53^{\prime}$ & 559 \\
\hline Acc. 25 & Balehalli & Hanagal & Haveri & Up land arecanut & $14^{0} .42^{\prime}$ & $75^{0} .41^{\prime}$ & 508 \\
\hline Acc. 26 & Hudelakoppa & Mundagod & Uttara Kannada & Rainfed paddy field & $14^{0} .48^{\prime}$ & $75^{0} .02^{\prime}$ & 492 \\
\hline Acc. 27 & Khalaghatagi & Khalaghatagi & Dharwad & Arecanut plantation & $15^{0} .10^{\prime}$ & $74^{0} .58^{\prime}$ & 470 \\
\hline Acc. 28 & Aralikoppa & Yellapura & Uttara Kannada & Forest & $14^{0} .59^{\prime}$ & $74^{0} .43^{\prime}$ & 487 \\
\hline Acc. 29 & $\begin{array}{l}\text { Kogilaban, } \\
\text { Dandeli }\end{array}$ & Haliyal & Uttara Kannada & Paddy field & $15^{0} .14^{\prime}$ & $74^{0} .37^{\prime}$ & 363 \\
\hline Acc. 30 & Usoda & Joida & Uttara Kannada & Low land arecanut & $15^{0} .18^{\prime}$ & $74^{0} .34^{\prime}$ & 434 \\
\hline Acc. 31 & Jagalpete & Joida & Uttara Kannada & Forest & $15^{0} .19^{\prime}$ & $74^{0} .30^{\prime}$ & 615 \\
\hline Acc. 32 & Londa & Khanapura & Belgaum & Paddy field & $15^{0} .29^{\prime}$ & $74^{0} .28^{\prime}$ & 602 \\
\hline Acc. 33 & Badagabailu & N.R. Pura & Chikkamagaluru & Rubber & $13^{0} .37^{\prime}$ & $75^{0} .29^{\prime}$ & 708 \\
\hline Acc. 34 & Mavinahalli & Chikkamagaluru & Chikkamagaluru & Acasia (Forest) & $13^{0} .14^{\prime}$ & $75^{0} .41^{\prime}$ & 1002 \\
\hline Acc. 35 & Arenoor & Chikkamagaluru & Chikkamagaluru & Paddy field & $13^{0} .17^{\prime}$ & $75^{0} .36^{\prime}$ & 679 \\
\hline Acc. 36 & Mundagod & Sringeri & Chikkamagaluru & Forest & $13^{0} .23^{\prime}$ & $75^{0} .18^{\prime}$ & 687 \\
\hline Acc. 37 & Agumbe & Thirthahalli & Shivamogga & Forest & $13^{0} .30^{\prime}$ & $75^{0} .06^{\prime}$ & 583 \\
\hline Acc. 38 & Ammadi & Koppa & Chikkamagaluru & Coffee plantation & $13^{0} .33^{\prime}$ & $75^{0} .20^{\prime}$ & 660 \\
\hline Acc. 39 & Gubgal & Mudigere & Chikkamagaluru & Coffee plantation & $13^{0} .15^{\prime}$ & $75^{0} .29^{\prime}$ & 742 \\
\hline
\end{tabular}


Table.2 Details of exploration and collection sites of Centella asiatica L. accessions in hill zone of Karnataka

\begin{tabular}{|c|c|c|c|c|}
\hline $\begin{array}{l}\text { Sl. } \\
\text { No. }\end{array}$ & District & Taluk & $\begin{array}{l}\text { Number of accessions } \\
\text { collected }\end{array}$ & Total \\
\hline \multirow[t]{3}{*}{1} & \multirow[t]{3}{*}{ Kodagu } & Virajpet & 02 & \multirow[t]{3}{*}{06} \\
\hline & & Madikeri & 02 & \\
\hline & & Somwarapet & 02 & \\
\hline 2 & Hassan & Sakaleshpura & 02 & 02 \\
\hline \multirow[t]{5}{*}{3} & \multirow{5}{*}{ Chikkamagaluru } & Mudigere & 03 & \multirow[t]{5}{*}{11} \\
\hline & & Sringeri & 02 & \\
\hline & & Koppa & 02 & \\
\hline & & N.R. Pura & 02 & \\
\hline & & Chikmagalur & 02 & \\
\hline \multirow[t]{4}{*}{4} & \multirow{4}{*}{ Shivamogga } & Thirthahalli & 02 & \multirow[t]{4}{*}{08} \\
\hline & & Hosanagara & 02 & \\
\hline & & Sagara & 02 & \\
\hline & & Sorabha & 02 & \\
\hline \multirow[t]{6}{*}{5} & \multirow[t]{6}{*}{ Uttara Kannada } & Siddapura & 02 & \multirow[t]{6}{*}{09} \\
\hline & & Sirsi & 02 & \\
\hline & & Mundagod & 01 & \\
\hline & & Yellapura & 01 & \\
\hline & & Haliyal & 01 & \\
\hline & & Joida & 02 & \\
\hline 6 & Haveri & Hanagal & 01 & 01 \\
\hline 7 & Belgaum & Khanapura & 01 & 01 \\
\hline 8 & Dharwad & Khalaghatagi & 01 & 01 \\
\hline Total & 08 & 22 & 39 & 39 \\
\hline
\end{tabular}

Fig.1 Map showing details of Centella asiatica L. accessions collection from hill zone of Karnataka

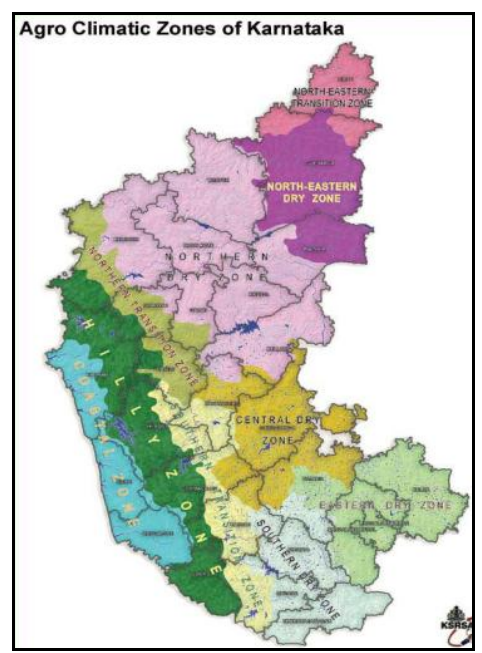


Table.3 Variability in plant growth habit, regenerability and leaf arrangement in Centella asiatica L. accessions.

\begin{tabular}{|c|c|c|c|}
\hline Accessions & Plant growth habit & Regeneration capacity & Leaf arrangement \\
\hline Acc. 1 & Erect & Good & Rosette \\
\hline Acc. 2 & Erect & Medium & Rosette \\
\hline Acc. 3 & Erect & Good & Rosette \\
\hline Acc. 4 & Erect & Good & Rosette \\
\hline Acc. 5 & Erect & Medium & Rosette \\
\hline Acc. 6 & Erect & Good & Rosette \\
\hline Acc. 7 & Erect & Good & Spreading \\
\hline Acc. 8 & Erect & Good & Spreading \\
\hline Acc. 9 & Erect & Poor & Rosette \\
\hline Acc. 10 & Erect & Medium & Rosette \\
\hline Acc. 11 & Erect & Medium & Rosette \\
\hline Acc. 12 & Erect & Good & Rosette \\
\hline Acc. 13 & Erect & Medium & Rosette \\
\hline Acc. 14 & Erect & Good & Rosette \\
\hline Acc. 15 & Erect & Medium & Rosette \\
\hline Acc. 16 & Prostate & Poor & Rosette \\
\hline Acc. 17 & Erect & Poor & Rosette \\
\hline Acc. 18 & Erect & Good & Rosette \\
\hline Acc. 19 & Erect & Good & Rosette \\
\hline Acc. 20 & Erect & Medium & Rosette \\
\hline Acc. 21 & Semi erect & Poor & Rosette \\
\hline Acc. 22 & Semi erect & Good & Rosette \\
\hline Acc. 23 & Semi erect & Poor & Rosette \\
\hline Acc. 24 & Erect & Good & Rosette \\
\hline Acc. 25 & Erect & Medium & Rosette \\
\hline Acc. 26 & Erect & Poor & Rosette \\
\hline Acc. 27 & Erect & Medium & Rosette \\
\hline Acc. 28 & Erect & Good & Rosette \\
\hline Acc. 29 & Erect & Medium & Rosette \\
\hline Acc. 30 & Erect & Good & Rosette \\
\hline Acc. 31 & Semi erect & Medium & Rosette \\
\hline Acc. 32 & Semi erect & Poor & Rosette \\
\hline Acc. 33 & Semi erect & Medium & Rosette \\
\hline Acc. 34 & Erect & Medium & Rosette \\
\hline Acc. 35 & Erect & Medium & Rosette \\
\hline Acc. 36 & Prostrate & Poor & Rosette \\
\hline Acc. 37 & Erect & Medium & Rosette \\
\hline Acc. 38 & Erect & Good & Rosette \\
\hline Acc. 39 & Prostate & Poor & Rosette \\
\hline Vallabh Medha* & Erect & Good & Spreading \\
\hline
\end{tabular}


Table.4 Variability in leaf morphology of Centella asiatica L. accessions

\begin{tabular}{|c|c|c|c|c|c|}
\hline \multirow[t]{2}{*}{ Accessions } & \multicolumn{5}{|l|}{ Leaf } \\
\hline & Size & Shape & Margin & Colour & Surface \\
\hline Acc. 1 & Medium & Orbicular & Crenate & Green & Glabraous \\
\hline Acc. 2 & Medium & Reniform & Dentate & Green & Glabraous \\
\hline Acc. 3 & Small & Orbicular & Crenate & Dark green & Glabraous \\
\hline Acc. 4 & Medium & Orbicular & Crenate & Dark green & Glabraous \\
\hline Acc. 5 & Medium & Orbicular & Dentate & Green & Glabraous \\
\hline Acc. 6 & Medium & Orbicular & Dentate & Dark green & Glabraous \\
\hline Acc. 7 & Large & Orbicular & Dentate & Light green & Glabraous \\
\hline Acc. 8 & Medium & Reniform & Dentate & Light green & Glabraous \\
\hline Acc. 9 & Medium & Orbicular & Crenate & Dark green & Glabraous \\
\hline Acc. 10 & Medium & Reniform & Dentate & Dark green & Glabraous \\
\hline Acc. 11 & Large & Reniform & Dentate & Dark green & Glabraous \\
\hline Acc. 12 & Large & Reniform & Dentate & Dark green & Glabraous \\
\hline Acc. 13 & Medium & Orbicular & Dentate & Dark green & Glabraous \\
\hline Acc. 14 & Medium & Orbicular & Dentate & Dark green & Glabraous \\
\hline Acc. 15 & Medium & Orbicular & Crenate & Light green & Glabraous \\
\hline Acc. 16 & Large & Orbicular & Dentate & Dark green & Glabraous \\
\hline Acc. 17 & Medium & Orbicular & Dentate & Green & Glabraous \\
\hline Acc. 18 & Medium & Reniform & Dentate & Light green & Glabraous \\
\hline Acc. 19 & Medium & Orbicular & Dentate & Light green & Glabraous \\
\hline Acc. 20 & Medium & Orbicular & Dentate & Light green & Glabraous \\
\hline Acc. 21 & Medium & Orbicular & Dentate & Dark green & Glabraous \\
\hline Acc. 22 & Medium & Reniform & Dentate & Dark green & Glabraous \\
\hline Acc. 23 & Medium & Reniform & Dentate & Green & Glabraous \\
\hline Acc. 24 & Medium & Orbicular & Dentate & Dark green & Glabraous \\
\hline Acc. 25 & Medium & Orbicular & Crenate & Dark green & Glabraous \\
\hline Acc. 26 & Large & Orbicular & Dentate & Light green & Glabraous \\
\hline Acc. 27 & Medium & Reniform & Dentate & Light green & Glabraous \\
\hline Acc. 28 & Medium & Orbicular & Dentate & Dark green & Glabraous \\
\hline Acc. 29 & Medium & Orbicular & Dentate & Light green & Glabraous \\
\hline Acc. 30 & Medium & Orbicular & Crenate & Dark green & Glabraous \\
\hline Acc. 31 & Medium & Orbicular & Dentate & Dark green & Glabraous \\
\hline Acc. 32 & Medium & Reniform & Dentate & Light green & Glabraous \\
\hline Acc. 33 & Medium & Orbicular & Crenate & Light green & Glabraous \\
\hline Acc. 34 & Medium & Orbicular & Crenate & Dark green & Glabraous \\
\hline Acc. 35 & Medium & Reniform & Crenate & Dark green & Glabraous \\
\hline Acc. 36 & Medium & Orbicular & Crenate & Dark green & Glabraous \\
\hline Acc. 37 & Medium & Orbicular & Crenate & Dark green & Glabraous \\
\hline Acc. 38 & Medium & Orbicular & Dentate & Light green & Glabraous \\
\hline Acc. 39 & Small & Reniform & Dentate & Light green & Glabraous \\
\hline Vallabh Medha* & Large & Orbicular reniform & Dentate & Greenish yellow & Glabraous \\
\hline
\end{tabular}

*Check variety 
Table.5 Variability in petiole and stolon characteristics of Centella asiatica L.accessions

\begin{tabular}{|c|c|c|c|c|c|c|}
\hline Accessions & $\begin{array}{l}\text { Petiole } \\
\text { length }\end{array}$ & $\begin{array}{l}\text { Petiole } \\
\text { thickness }\end{array}$ & $\begin{array}{l}\text { Petiole } \\
\text { pigmentation }\end{array}$ & $\begin{array}{l}\text { Stolon } \\
\text { colour }\end{array}$ & $\begin{array}{l}\text { Flower } \\
\text { colour }\end{array}$ & $\begin{array}{l}\text { Stolon } \\
\text { texture }\end{array}$ \\
\hline Acc. 1 & Medium & Thin & Light pink & Greenish pink & Pink & Soft \\
\hline Acc. 2 & Medium & Thick & Pink & Greenish pink & Pink & Soft \\
\hline Acc. 3 & Long & Thick & Pink & Greenish pink & Pink & Hard \\
\hline Acc. 4 & Medium & Thin & Light pink & Greenish pink & Pink & Soft \\
\hline Acc. 5 & Medium & Thin & Pink & Greenish pink & Pink & Soft \\
\hline Acc. 6 & Medium & Thin & Pink & Light pink & Pink & Soft \\
\hline Acc. 7 & Long & Thick & Pink & Light pink & Light pink & Hard \\
\hline Acc. 8 & Medium & Thin & Pink & Pink & pink & Hard \\
\hline Acc. 9 & Medium & Thin & Light pink & Greenish pink & Light pink & Soft \\
\hline Acc. 10 & Medium & Thick & Pink & Pink & Pink & Hard \\
\hline Acc. 11 & Medium & Thick & Light pink & Greenish pink & Pink & Hard \\
\hline Acc. 12 & Long & Thin & Pink & Pink & Pink & Hard \\
\hline Acc. 13 & Medium & Thin & Pink & Greenish pink & Light pink & Soft \\
\hline Acc. 14 & Long & Thin & Pink & Greenish pink & Dark pink & Soft \\
\hline Acc. 15 & Medium & Thin & Pink & Greenish pink & Pink & Soft \\
\hline Acc. 16 & Medium & Thin & Pink & Greenish pink & Pink & Soft \\
\hline Acc. 17 & Medium & Thin & Light pink & Light pink & Greenish pink & Soft \\
\hline Acc. 18 & Long & Thin & Light Pink & Light pink & Pink & Soft \\
\hline Acc. 19 & Medium & Thin & Dark pink & Dark pink & Light pink & Soft \\
\hline Acc. 20 & Medium & Thin & Light pink & Greenish pink & Light pink & Soft \\
\hline Acc. 21 & Long & Thin & Light pink & Light pink & Pink & Soft \\
\hline Acc. 22 & Medium & Thick & Pink & Dark pink & Pink & Soft \\
\hline Acc. 23 & Long & Thin & Light pink & Dark pink & Pink & Soft \\
\hline Acc. 24 & Medium & Thick & Light pink & Greenish pink & Pink & Hard \\
\hline Acc. 25 & Medium & Thick & Light pink & Greenish pink & Pink & Hard \\
\hline Acc. 26 & Medium & Thin & Light pink & Pink & Pink & Soft \\
\hline Acc. 27 & Medium & Thin & Greenish pink & Greenish pink & Greenish pink & Soft \\
\hline Acc. 28 & Medium & Thin & Pink & Pink & Pink & Soft \\
\hline Acc. 29 & Medium & Thick & Pink & Pink & Pink & Hard \\
\hline Acc. 30 & Medium & Thick & Pink & Light pink & Pink & Hard \\
\hline Acc. 31 & Long & Thick & Pink & Dark pink & Pink & Hard \\
\hline Acc. 32 & Medium & Thick & Pink & Greenish pink & Pink & Hard \\
\hline Acc. 33 & Medium & Thin & Light pink & Greenish pink & Pink & Soft \\
\hline Acc. 34 & Medium & Thin & Light pink & Dark pink & Pink & Soft \\
\hline Acc. 35 & Medium & Thick & Light pink & Pink & Pink & Hard \\
\hline Acc. 36 & Small & Thick & Light pink & Light pink & Pink & Soft \\
\hline Acc. 37 & Medium & Thin & Greenish pink & Greenish pink & Pink & Soft \\
\hline Acc. 38 & Medium & Thin & Greenish pink & Greenish pink & Greenish pink & Soft \\
\hline Acc. 39 & Small & Thin & Pink & Greenish pink & Pink & Soft \\
\hline $\begin{array}{l}\text { Vallabh } \\
\text { Medha* }\end{array}$ & Long & Thin & Dark pink & Light pink & Pink & Soft \\
\hline
\end{tabular}

*Check variety 
Table.6 Summary statistics of morphological characterization of Centella asiatica L. accessions used in the present study

\begin{tabular}{|c|c|c|c|c|}
\hline $\begin{array}{l}\text { SI. } \\
\text { No }\end{array}$ & Character & Phenotype & $\begin{array}{l}\text { Number of } \\
\text { accessions }\end{array}$ & $\begin{array}{l}\text { Percentage } \\
\text { of distribution }\end{array}$ \\
\hline \multirow[t]{3}{*}{1} & \multirow[t]{3}{*}{ Plant growth habit } & Erect & 31 & 77.50 \\
\hline & & Semi erect & 06 & 15.00 \\
\hline & & Prostrate & 03 & 07.50 \\
\hline \multirow[t]{3}{*}{2} & \multirow{3}{*}{ Regenerability } & Good & 16 & 40.00 \\
\hline & & Medium & 15 & 37.50 \\
\hline & & Poor & 09 & 22.50 \\
\hline \multirow[t]{2}{*}{3} & \multirow[t]{2}{*}{ Leaf arrangement } & In rosettes & 37 & 92.50 \\
\hline & & Spreading & 03 & 07.50 \\
\hline \multirow[t]{3}{*}{4} & \multirow[t]{3}{*}{ Leaf size } & Small & 02 & 05.00 \\
\hline & & Medium & 32 & 80.00 \\
\hline & & Large & 06 & 15.00 \\
\hline \multirow[t]{3}{*}{5} & \multirow[t]{3}{*}{ Leaf shape } & Orbicular & 27 & 67.50 \\
\hline & & Reniform & 12 & 30.00 \\
\hline & & Orbicular-reniform & 01 & 2.50 \\
\hline \multirow[t]{3}{*}{6} & \multirow[t]{3}{*}{ Leaf margin } & Crenate & 12 & 30.00 \\
\hline & & Dentate & 28 & 70.00 \\
\hline & & Wavy & - & 0.00 \\
\hline \multirow[t]{4}{*}{7} & \multirow{4}{*}{ Leaf colour } & Light green & 13 & 32.50 \\
\hline & & Dark green & 21 & 52.50 \\
\hline & & Green & 05 & 12.50 \\
\hline & & Greenish yellow & 01 & 2.50 \\
\hline \multirow[t]{2}{*}{8} & \multirow[t]{2}{*}{ Leaf surface } & Glabrous & 40 & 100.00 \\
\hline & & Pubescent & - & 00.00 \\
\hline \multirow[t]{3}{*}{9} & \multirow[t]{3}{*}{ Petiole length } & Small & 02 & 05.00 \\
\hline & & Medium & 29 & 72.50 \\
\hline & & Long & 09 & 22.50 \\
\hline \multirow[t]{2}{*}{10} & \multirow{2}{*}{ Petiole thickness } & Thick & 14 & 35.00 \\
\hline & & Thin & 26 & 65.00 \\
\hline \multirow[t]{4}{*}{11} & \multirow{4}{*}{$\begin{array}{l}\text { Petiole pigmentation at } \\
\text { the base }\end{array}$} & Light pink & 16 & 40.00 \\
\hline & & Dark pink & 02 & 05.00 \\
\hline & & Greenish pink & 03 & 07.50 \\
\hline & & Pink & 19 & 47.50 \\
\hline \multirow{4}{*}{12} & \multirow{4}{*}{ Stolon colour } & Light pink & 08 & 20.00 \\
\hline & & Dark pink & 05 & 12.50 \\
\hline & & Greenish pink & 20 & 50.00 \\
\hline & & Pink & 07 & 17.50 \\
\hline \multirow{4}{*}{13} & Flower colour & Light pink & 05 & 12.50 \\
\hline & & Dark pink & 01 & 02.50 \\
\hline & & Greenish pink & 03 & 07.50 \\
\hline & & Pink & 31 & 77.50 \\
\hline 14 & Texture of stolon & Hard & 13 & 32.50 \\
\hline & & Soft & 27 & 67.50 \\
\hline
\end{tabular}


Plate.1 Variation in leaf shapes in ecotypes of Centella asiatica L. accessions

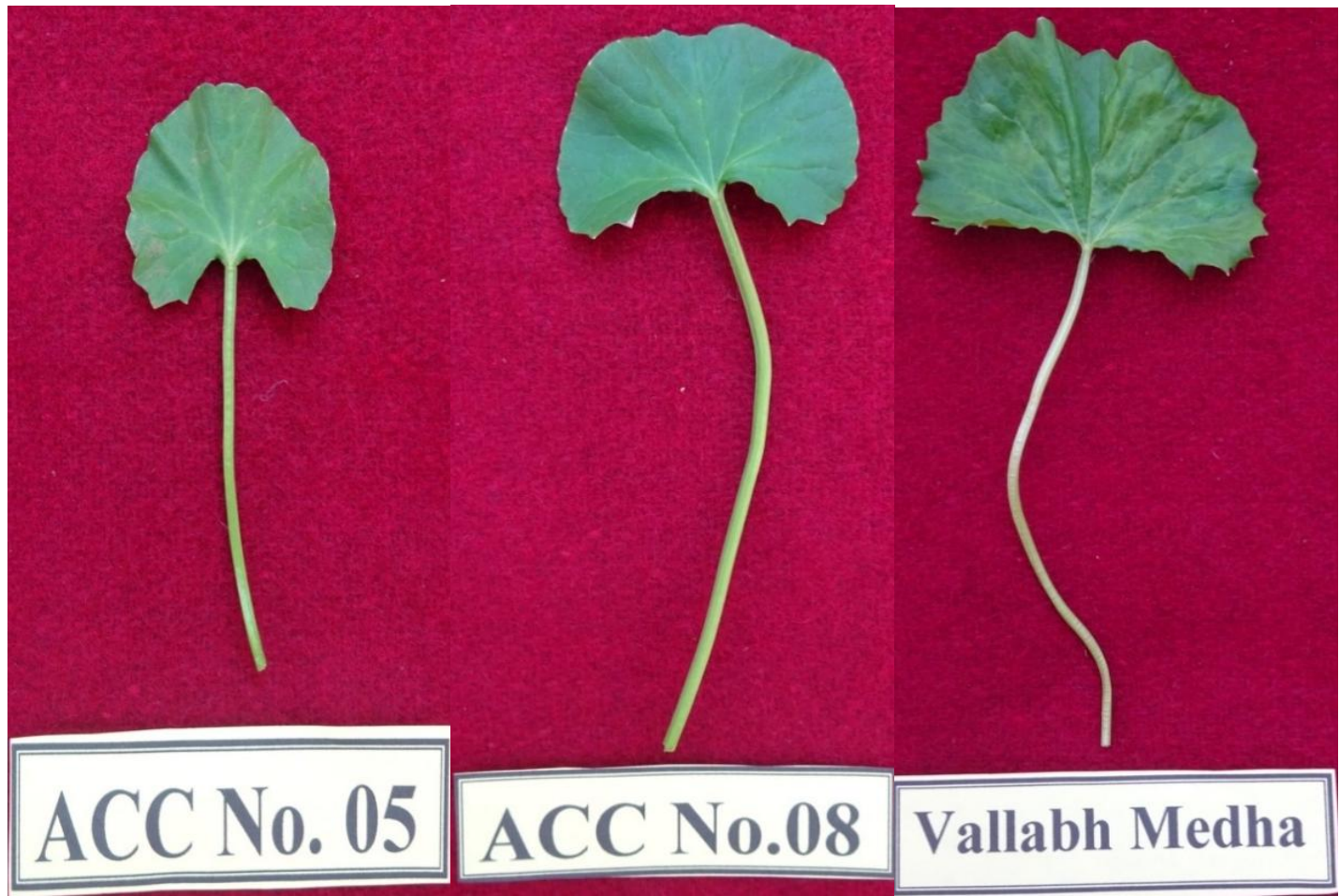

Orbicular

Reniform

Orbicular reniform

Plate.2 Variation in leaf margin in ecotypes of Centella asiatica L. accessions

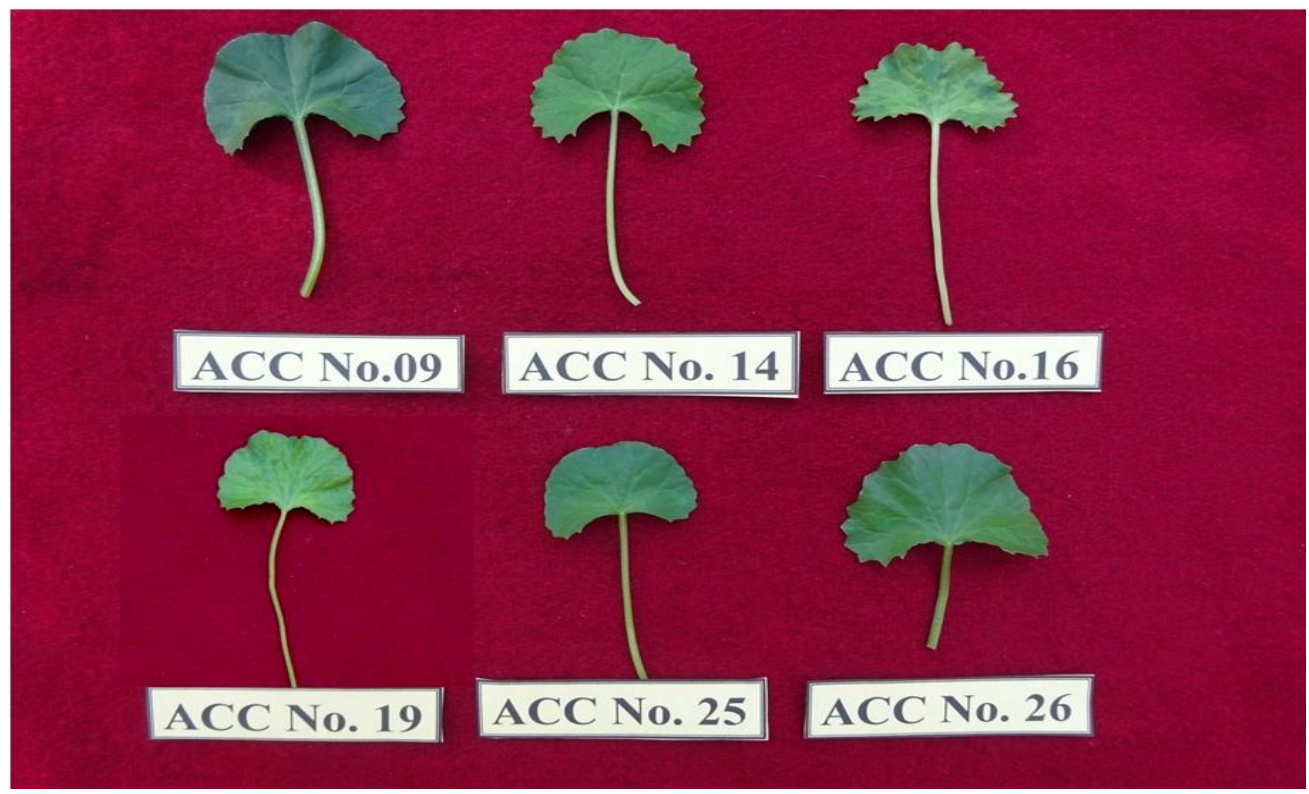



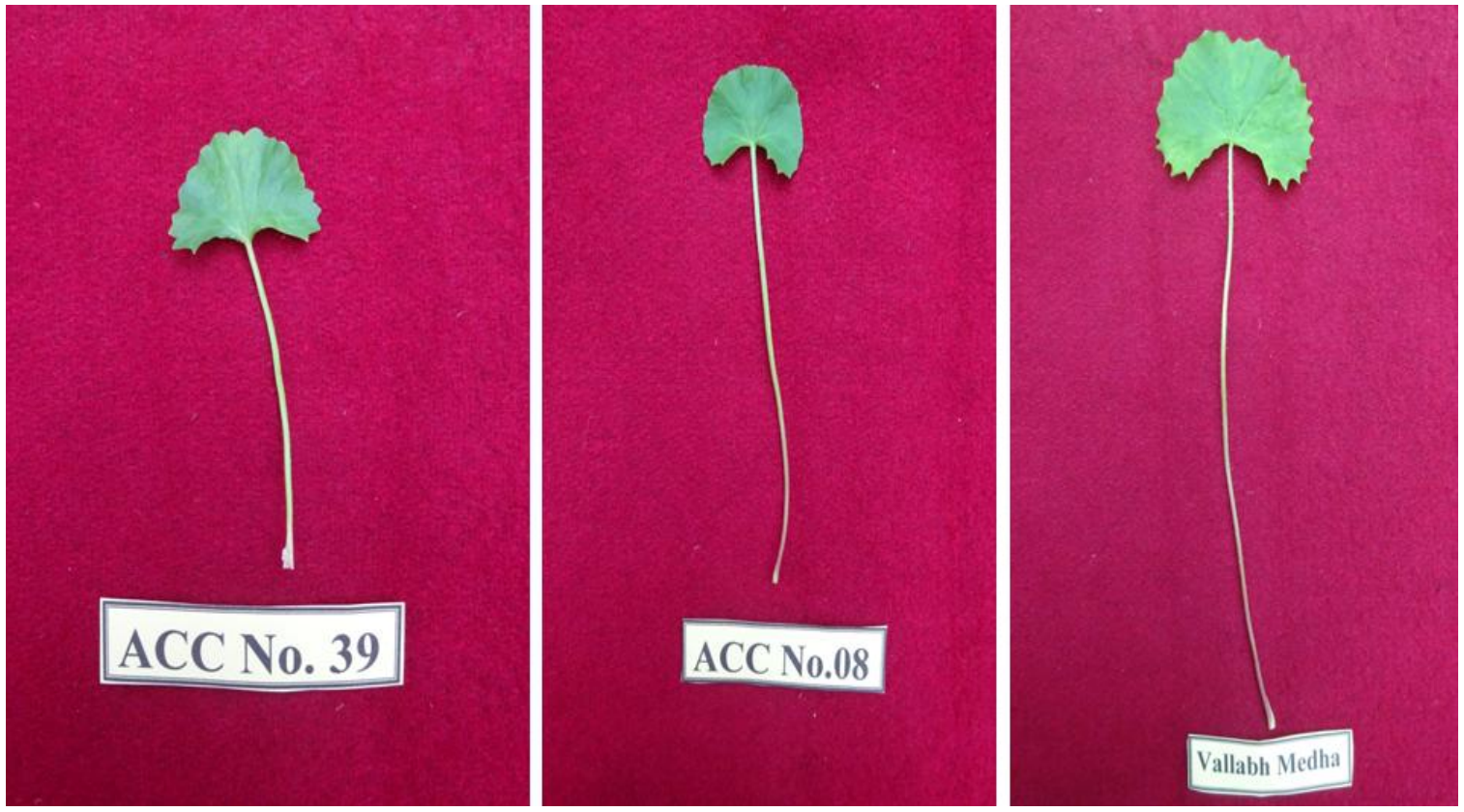

Plate.4 Variation in petiole pigmentation at the base in ecotypes of Centella asiatica L. accessions
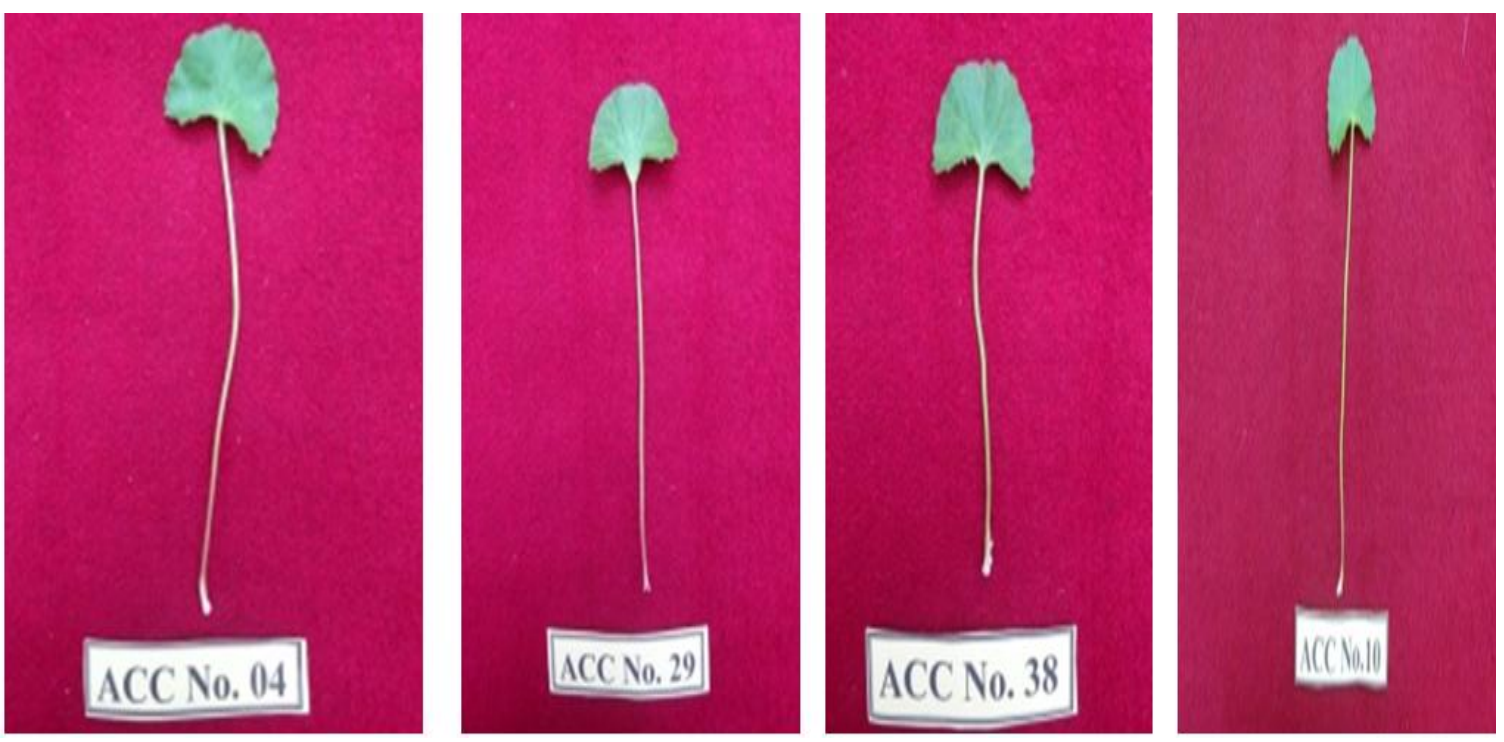

Similar variations in leaf size were reported by Prasad et al., (2014) in Centella asiatica and Tripathi et al., (2012) in brahmi.
Wide phenotypic variations were recorded among the accessions studied for leaf shape (Plate 1) and majority of the accessions (27) had orbicular shape (Acc. 1, 3, 4, 5, 6, 7, 9, 13, 
$14,15,16,17,19,20,21,24,25,26,28,29$, $30,31,33,34,36,37$ and 38). While, the accessions such as Acc. 11, 2, 8, 10, 12, 18, $22,23,27,32,35$ and 39 had reniform shape except Vallabh Medha which had orbicular reniform shape. The presence of characteristic leaf shape is a key morphological marker for identification and characterization of a particular accession. The variations in leaf shape could be ascribed to genetic makeup of the accessions. Variation in leaf shapes were observed by Prasad et al., (2014) in Centella asiatica and Roshni et al., (2014) in brahmi.

Among the accessions, marked variations in leaf margin (Plate 2) were observed. Most of the accessions (28) had dentate margin (Acc. $2,5,6,7,8,10,11,12,13,14,16,17,18,19$, 20, 21, 22, 23, 24, 27, 26, 28, 29, 31, 32, 38, 39 and Vallabh Medha) and rest of the accessions (12) exhibited crenate margin(Acc. $1,3,4,9,15,25,30,33,34,36,37$ and 35). The manifestation of characteristic leaf margin is also a prime morphological marker for identification and categorization of a particular accession. The variations in leaf margin among the accessions is due to differences in their genetic makeup. Similarly, the variations in leaf margin among the accessions were reported by Prasad et al., (2014) in Centella asiatica andMalav et al., (2015) in holy basil.

The leaf colouration was dark green colour in 21 accessions (Acc. 3, 4, 6, 9, 10, 11, 12, 13, $14,16,21,22,24,25,28,30,31,34,35,36$ and 37). The accessions such as Acc.1, 2, 5, 17 and 23 produced green leaves while, rest of the accessions possessed light green colour except Vallabh Medha which had greenish yellow leaves. The variations in leaf colour could be attributed to genetic makeup of the accessions as well as the environmental conditions, as these interact and the resultant colour depends on these two. However, under a given set of conditions, any variation could be attributed to the genetic constitution of the plant. Phenotypic variability for the leaf colour among the accessions were reported in Centella asiatica (Mathur et al., 2003); brahmi (Tripathi et al., 2012) and Aloe vera (Kaur and Saggoo, 2010).

Leaf surface among the accessions did not vary markedly and all the accessions were of glabrous type. The petiole length among the accessions showed remarkable variations. Long, medium and short petioles were recorded in nine, 29 and two accessions, respectively. The accessions Acc.03, 7, 12, 14, 18, 21, 23, 31 and Vallabh Medha were long petioled. Medium to long petioled accessions are desirable as it contributes to higher herbage. The petiole length (Plate 3) is genetically controlled and the variations among the accessions is due to differences in the genetic makeup. Variations in petiole length among the accessions were also noticed by Padmalatha and Prasad (2008) in Centella asiatica.

The thick petioled accessions were Acc. 2, 3, $7,10,11,22,24,25,29,30,31,32,35$ and 36. While, rest of the accessions possessed thin petiole. The variations in petiole thickness could be attributed to differences in their genetic makeup.

Wide variations for pigmentation at the base of petiole were noticed (Plate 4), which varied from light pink, pink, dark pink and greenish pink. Among these, pink pigmentation was found to be a dominant trait (15 accessions), followed by light pink (16 accessions), while, greenish pink and dark pink pigmentations were recorded in three (Acc. 27, 37 and 38) and two accessions (Acc. 19 and Vallabh Medha), respectively. Dark pink to pink pigmentation at petiole base is due to differential distribution of anthocyanin pigments and could be attributed to antioxidant properties. The expression of 
pigmentation in petiole would aid in characterization of unique accessions, as it serves as colour marker. The variation in pigmentation in petiole base is attributed to differences in the genetic makeup of accessions. Roshni et al., (2014) reported pink coloration in stems of certain accessions of brahmi and Kaur and Saggoo (2010) in spine colour among North Indian Aloe vera accessions.

The stolon colour was dark pink (Acc. 19, 22, 23, 31 and 34) and pink in Acc. 8, 10, 12, 26, 28, 29 and 35. While, it was light pink in Acc. $6,7,17,18,21,30,36$ and Vallabh Medha. All remaining accessions had greenish pink stolon. The manifestation of stolon colour in accessions would help in identification and characterization of unique accessions as it serves as colour marker and also attributed to antioxidant properties. The differences in the stolon colour of accessions can be attributed to variations in genetic makeup and differential distribution of anthocyanin pigments. Similar variations among the accessions of brahmi for stem colour have been reported (Roshni et al., 2014).

Among 40 accessions studied, Acc.14 alone recorded dark pink flower. The light pink flowers were noticed in five accessions (Acc.7, 9, 13, 19 and 20) and it was greenish pink in Acc. 17, 27 and 38. All remaining accessions had pink coloured flowers. Although difference in flower colour is genetically controlled it does interact with the environmental conditions more particularly light intensity and duration. But the difference in the flower colour in any given set of environmental conditions is always due to differential genetic makeup in various accessions. Roshni et al., (2014) in brahmi reported variation in flower colours in different accessions.

Thirteen accessions (Acc. 3, 7, 8, 10, 11, 12,
24, 25, 29, 30, 31, 32 and 35) had hard texture in the stolon. All other accessions had soft texture. The variation in stolon texture shall be attributed to differences in their genetic makeup.

\section{References}

Kaur, R. and Saggoo, M.I.S., 2010.Evaluation and improvement of germplasm of Aloe vera L. from North India.Ph.D. Thesis, Punjabi University, Patiala (India).

Kundu, S., Haque, S.M. and Ghosh, B., 2015.Comparative analysis of bioactive compounds in different habitat of Centella asiatica (L.) Urban: Application for in vitro clonal propagation of elite ecotype. J. Appl. Pharm. Sci., 5(2): 30-36.

Lal, R.K. Gupta, P. and Dubey, B. K.,2017. Genetic variability and associations in the accessions of mandukaparni. Ind. Crops and Prod., 96:173-177.

Malav, P., Pandey, A., Bhatt, K. C., Krishnan, S. G.and Bisht. I. S., 2015.Morphological variability in Holy basil (Ocimum tenuiflorum L.) from India. Genet. Resour. Crop Evolution., 62(8): 1245-1256.

Mathur, S., Sharma, S. and Kumar, S., 2003. Description of variation in the Indian accessions of the medicinal plant Centella asiatica (L.) Urban.Plant Genetic Resources Newsletter, 135: 4752.

Mukherjee, P. K. and Constance, L., 1993. Umbelliferae (Apiaceae)of India. Oxford \& IBH Publishing Company Pvt. Ltd., New Delhi.

Padmalatha, K. and Prasad, M.N.V., 2008. Genetic diversity in Centella asiatica (L.) Urb.,a memory enhancing neutraceutical herb using RAPD Markers. Med.and Aromat. Plant Sci. Biotech.,2(2),90-95. 
Prasad, A., Dhawana, S.S., Mathura, A.K., Om Prakash, Gupta, M.M., Verma, R.K., Lal, R.K. and Mathura, A., 2014. Morphological, chemical and molecular characterization of Centella asiatica germplasms for commercial cultivation in the Indo-Gangetic plains. Natural Product Communications, 9 (6): 779784.

Roshni, L.S., Gangaprasad, A. and Siril, E.A., 2014. Evaluation of variability in Bacopamonnieri (L.) Pennell using morphological and biochemical markers.Int. J. Applied Res. Natural Products. 7 (2):25-31.

Schaneberg, B.T., Mikell, J.R., Bedir, E. and Khan,I.A., 2003. An improved HPLC method for quantitative determination of six triterpenes in Centella asiatica extracts and commercial products. Pharmazie, 58(6):381-384.
Singh, J., Sangwan, R.S., Gupta, S., Saxena. S. and Sangwan, N.S., 2015.Profiling of triterpenoid saponin content variation in different chemotypic accessions of Centella asiatica L. Plant Genetic Resources, 13: 176-179.

Thapa B., Mahato, S. K., Khawas, T., Chettri, B., Vineeta and Ghimiray T.S., 2016. Morphological and genetic variability studies in Centella asiatica of Darjeeling hills. J. Agric. Technolol., 3 (2): 12-17.

Tripathi, N., Chouhan, D.S., Saini, N. and Tiwari, S., 2012. Assessment of genetic variations among highly endangered medicinal plant Bacopamonnieri (L.) from Central India using RAPD and ISSR analysis. Biotech., 2:327-336.

\section{How to cite this article:}

Ravi, C. S., K. Umesha, K. HimaBindu, G. Raviraja Shetty, B. S. Sreeramu, B. Fakrudin, A. P. MallikarjunaGowda and Anil Kumar, G. S. 2019. Collection and Morphological Variability in Ecotypes of Indian Pennywort (Centella asiatica L.) of Hill Zone of Karnataka, India. Int.J.Curr.Microbiol.App.Sci. 8(09): 994-1008. doi: https://doi.org/10.20546/ijcmas.2019.809.117 